(2) Open Access Full Text Article

\title{
Cysteamine hydrochloride eye drop solution for the treatment of corneal cystine crystal deposits in patients with cystinosis: an evidence-based review
}

This article was published in the following Dove Press journal:

Clinical Ophthalmology

\author{
Achini K Makuloluwa \\ Fatemeh Shams \\ Tennent Institute of Ophthalmology, \\ Gartnavel General Hospital, \\ Glasgow, UK
}

\begin{abstract}
Cystinosis is a rare, autosomal recessive disorder leading to defective transport of cystine out of lysosomes. Subsequent cystine crystal accumulation can occur in various tissues, including the ocular surface. This review explores the efficacy of cysteamine hydrochloride eye drops in the treatment of corneal cystine crystal accumulation and its safety profile.
\end{abstract}

Keywords: cysteine, CTNS gene, cystaran, cystadrops

\section{Introduction to epidemiology, etiology, and pathophysiology of cystinosis}

Cystinosis is a rare disease affecting lysosomal storage. ${ }^{1,2}$ Normally, cystine - a disulfide amino acid - is transported out of lysosomes into the cytoplasm via cystinosin with the aid of $\mathrm{H}^{+}$ions. ${ }^{3,4}$ Cystinosin is a 367 -amino acid protein with seven transmembrane domains. It is coded for by the CTNS gene of 12 exons spanning 23 $\mathrm{kb}$ on chromosome $17 \mathrm{p} 13.2$ discovered in $1998 .^{2-5}$ Patients with infantile or nephropathic cystinosis, the commonest subgroup of cystinosis, develop renal symptoms by 6-12 months of age in addition to the extra-renal complications. ${ }^{2,3}$ Juvenile or adolescent cystinosis has a later onset and is a milder form of the disease with effects on the kidneys and the eyes. ${ }^{2,3}$ Ocular cystinosis merely shows evidence of ophthalmic crystal deposition. ${ }^{2,3}$

Cystinosis is an autosomal recessive disorder leading to failure of cystine transport out of the lysosomes. ${ }^{2,4}$ The $57-\mathrm{kb}$ deletion of the CTNS gene is the commonest mutation encountered in the European population with infantile cystinosis, where $76 \%$ are either homozygous or heterozygous with this mutation. ${ }^{5-7}$ Moreover, this deletion affects $C A R K L$ and TRPVI genes. The CARKL gene is involved in sedoheptulose phosphorylation in the pentose phosphate pathway, and sedoheptulose levels in blood and urine can be used to screen families carrying this mutation. ${ }^{5,8}$ Many other mutations as well as no mutations have been reported to give rise to this condition..$^{9-12}$ In contrast, this commonly reported 57-kb deletion has not been encountered in non-European countries, such as Egypt and Turkey, highlighting the array of varied mutations within different population groups. ${ }^{13-15}$ Typically, the mutation involved in infantile cystinosis leads to complete loss of cystine transport protein, whereas that involved in adolescent and ocular cystinosis leads to a reduction in the functioning cystine transport system. ${ }^{16,17}$ Crystal production from a defective or diminished transport system and subsequent accumulation of cystine leads to a variety of phenotypes. In vivo studies have shown that lysosomal cystine leads to apoptosis of cells via cysteinylation or mixed disulfide formation. ${ }^{18,19}$
Correspondence: Achini K Makuloluwa Tennent Institute of Ophthalmology, Gartnavel General Hospital, Glasgow, GI2 OYN UK

Email achini.makuloluwa@gmail.com 
Epidemiological studies dating back to 1959 show that consanguinity plays a role in the incidence of cystinosis and it tends to vary among different population groups. However, its highest incidence was reported to be 1 in 3,613 and 1 in 25,989 live births in a cohort of Pakistani-origin population living in West Midlands, UK, and Brittany, France, respectively. ${ }^{20-29}$

Renal compromise leads to hypophosphatemic rickets and growth retardation. ${ }^{30}$ Extra-renal sequelae include hypothyroidism secondary to thyroid gland fibrosis (75\%), male hypergonadotropic hypogonadism secondary to testicular fibrosis and atrophy (74\%), myopathy (50\%), swallowing difficulties $(60 \%)$, pulmonary failure secondary to muscle weakness and atrophy (69\%), diabetes mellitus requiring insulin (24\%), hypercholesterolemia (33\%), vascular calcifications (31\%), and cerebral calcifications $(22 \%))^{4,31}$

Ocular abnormalities include both anterior and posterior segment pathology. ${ }^{32,33}$ Cystine crystals are deposited in all layers of the cornea - mostly, the stroma and, typically, in the peripheral cornea - by 16 months of age. ${ }^{34}$ Consequences of crystal deposition include photophobia, punctate keratopathy, filamentary keratitis, recurrent epithelial erosions causing pain, visual impairment, and scarring. ${ }^{34,35}$ Further, these crystals aggregate in the conjunctiva, iris, and ciliary body with further complications of band keratopathy, corneal neovascularization, posterior synechiae, pupillary block, and secondary glaucoma. ${ }^{35}$ Crystal accumulation in the posterior segment, including the retina, choroid, and optic nerve, presents as pigmentary retinopathy, reduced color vision, reduced night vision, reduced contrast sensitivity, field loss, and central vision loss. ${ }^{4,35-37}$ Moreover, papilledema has been reported secondary to raised intracranial pressure. ${ }^{35}$

A study carried out, in 1983, among 205 patients with cystinosis showed a median survival time of 8.5 years. ${ }^{38}$ The average time to end-stage renal failure was approximately 9 years. ${ }^{38,39}$ Subsequent studies after the introduction of oral cysteamine (beta-mercapto-ethyl-amine) or Cystagon, approved by the Food and Drug Administration (FDA) in 1994, showed more hopeful outcomes. ${ }^{4}$ A study from 2007 among 100 patients enrolled at the National Institutes of Health with nephropathic cystinosis and receiving oral cysteamine found that renal transplantation usually occurred by a mean age of 12.3 years and death occurred at a mean age of 28.5 years in one-third of their study population. ${ }^{4}$ Similar results were seen in a European study from 2011 of a cohort of patients with infantile cystinosis, where renal replacement therapy was only required at a mean age of 12.8 years. ${ }^{40}$ Studies showed that this increase in time to renal failure was contributed to by the introduction of oral cysteamine therapy. ${ }^{40-42}$ Furthermore, renal transplants were shown to have better prognosis in patients with cystinosis, where graft survival was $94 \%$ at 5 years and $86.5 \%$ at 10 years..$^{40,42,43}$ Moreover, a study showed that patients with cystinosis in developing countries, where only $54 \%$ of their study population received oral cysteamine, had their median time to kidney failure reduced by 6.4 years compared to a study population in developed countries, where all patients received oral cysteamine. ${ }^{44}$ Once oral cysteamine became more accessible in the developing world, this discrepancy in time to renal failure reduced to 3.8 years. ${ }^{44}$

Due to the rich vascular supply of choroid and retinal pigment epithelium, oral cysteamine showed a benefit in treating retinal crystal deposition. ${ }^{36}$ However, such success was not evident in treating anterior segment ocular surface cystine accumulation, and a case report also documented recurrence of cystine crystals on donor cornea following penetrating keratoplasty for cystinosis. ${ }^{45,46}$ With regard to corneal changes, a study on $\mathrm{CTNS}^{(--)}$knockout mice showed an increase in crystal deposition with time. Treatment with cysteamine drops showed a significant reduction in crystal deposition in the treated eyes with time (15\% rise) compared to untreated eyes (173\% rise). ${ }^{47}$

The aim of this article is to review the efficacy and the safety of topical cysteamine hydrochloride drops in patients with cystinosis who have corneal cystine crystal deposition.

\section{Review of pharmacology, mode of action, and pharmacokinetics of cysteamine hydrochloride eye drop solution}

Cysteamine is a free aminothiol which enters lysosomes. ${ }^{4}$ A disulfide reaction with cystine results in cysteine and cysteine-cysteamine compounds that can exit lysosomes via a different route to the conventional cystinosin pathway.,48

The current cysteamine drops have several drawbacks and limitations. The hydrophilic cysteamine eye drops have poor penetration through the lipophilic corneal epithelium; therefore, lesser amount of drug reaches the hydrophilic stroma, where cystine crystals are vastly deposited. ${ }^{49}$ This was evident in a study that compared the pharmacokinetics of cysteamine through a full-thickness cornea and de-epithelialized cornea and found cysteamine penetration was greater through a de-epithelialized cornea. ${ }^{49}$ In addition, as with most topical drops, cysteamine drops are known to have a brief residence time on the ocular surface, thus requiring regular administration of treatment - up to 12 times per day and likely leads 
to poor compliance by patients. ${ }^{49}$ Moreover, it is unstable in aqueous solution at room temperature as cysteamine oxidizes to its inactive form cystamine; therefore, it needs to be stored at $-20^{\circ} \mathrm{C}$ and kept refrigerated once opened. ${ }^{49}$ The SigmaTau Pharmaceuticals product Cystaran, $0.44 \%$ cysteamine with benzalkonium chloride (BAC) $0.65 \%$, received FDA approval in October 2012, and was commercially available from May 2013 - nonetheless, costing US\$875 per $15 \mathrm{~mL}$ bottle. ${ }^{50}$ Due to the unstable nature of the drug, it has a limited shelf life of 1 week..$^{50}$

A Franz-type diffusion model was used to study penetration of $0.44 \%$ cysteamine in the donor compartment through porcine cornea. ${ }^{49}$ Cysteamine penetration was analyzed using the receptor solution and was only detected after 60 minutes, which was deemed to be due to its poor penetrance and instability. ${ }^{49}$ Furthermore, corneal penetration of cysteamine was found to be poor at $\mathrm{pH} 4.2$, but was enhanced at $\mathrm{pH} 7.4$ because cysteamine existed in both unionized and amphiphilic forms at $\mathrm{pH} 7.4 .^{49}$ It was assumed that, in vivo, lacrimal fluid with $\mathrm{pH} 7.4$ would result in a $\mathrm{pH}$ shift of the unbuffered cysteamine solution, allowing greater corneal penetration. ${ }^{49}$ However, at $\mathrm{pH} 7.4$ and at increased temperatures, these unionized thiol groups were more prone to oxidation. ${ }^{49}$

The study group further examined excipients and discovered that disodium edetate (EDTA) did not aid cysteamine penetration at either $\mathrm{pH} 4.2$ or $7.4 .^{49}$ In contrast, BAC enhanced cysteamine penetration by ten times after 5 hours, and the initial time at which the receptor compartment identified cysteamine then reduced to 1 hour from 4 hours. ${ }^{49}$ Furthermore, BAC reduced the surface tension, allowing the gel more dispersive power on the ocular surface. ${ }^{51}$ Moreover, alpha-cyclodextrin allowed 20 times greater penetrance of cysteamine at a concentration of $5.5 \%{ }^{49}$ The time to identification of cysteamine in the receptor compartment still remained at 1 hour. ${ }^{49} \mathrm{BAC}$ and alpha-cyclodextrin did not alter the stability of cysteamine. ${ }^{49}$ In contrast, EDTA - on its own or when combined with sodium phosphate - had a protective effect on cysteamine stability. ${ }^{49}$ It did not negatively affect the enhanced permeability effect of alpha-cyclodextrin. ${ }^{49}$ The aforementioned findings have yet to be investigated in vivo. ${ }^{49}$

The bioavailability of a drug on the ocular surface can be optimized with gels, fornix inserts, punctual plus, subconjunctival inserts, and contact lenses. ${ }^{52}$ In vitro studies attempted to investigate some of the above vehicles as a form of delivering cysteamine to the ocular surface, with the aim of ultimately improving patient compliance and their quality of life.
A study from 2008 investigated Hi-Tech Pharmaceutical's preparation of cysteamine hydrochloride $0.55 \%$, monosodium phosphate, disodium EDTA, and BAC, made viscous with hydroxypropylmethylcellulose (HPMC).$^{53}$ Increased viscosity was seen with HPMC concentrations of $0.5 \%-1.5 \%$; however, the active drug release was better at a concentration of $1.0 \%$ compared to HPMC $1.5 \% .^{53}$ The stability of this gel cysteamine formulation decreased with time over 1 year at room temperature, but remained constant at $4{ }^{\circ} \mathrm{C} .{ }^{53} \mathrm{~A}$ study from 2010 also used Carbomer 934 to prepare a cysteamine hydrochloride gel and showed continuous drug release in its active form. ${ }^{54}$ A study from 2016 considered hydrogel formulations for cysteamine hydrochloride $0.55 \%$ and $\mathrm{BAC}$ as a preservative. ${ }^{51}$ Hydrogels can be pseudoplastic, transparent, and bioadhesive, thereby permitting prolonged drug release and, thus, optimizing bioavailability and compliance through less frequent drug administration. ${ }^{51}$ Carbomer 974 $(0.25 \%)$ and xanthan $(0.3 \%)$ gels were proven to be of poor stability. ${ }^{51}$ In contrast, sodium hyaluronate $(0.3 \%)$, carbomer $934(0.3 \%)$, and hydroxyethyl cellulose $(0.8 \%)$ dispensed cysteamine over 45-50 minutes. ${ }^{51}$ However, only sodium hyaluronate $(0.3 \%)$ showed long-term stability and was greatest when dispensed in ampoules. ${ }^{51}$

In vitro studies showed that contact lenses saturated with $50 \mathrm{mg} / \mathrm{mL}$ cysteamine can result in six times higher bioavailability, but the drug-dispensing time was short due to the low molecular weight of cysteamine and its hydrophilic properties. ${ }^{52}$ In contrast, 1-Day Acuvue ${ }^{\circledR}$ TrueEye with vitamin E $10.22 \%$ and Acuvue Oasys contact lenses combined with vitamin E 19.14\% extended cysteamine drug-release time to 25 minutes and 3 hours, respectively, from 10 minutes. ${ }^{52}$ Moreover, vitamin $\mathrm{E}$ had a protective role from oxidation of cysteamine. ${ }^{52}$ It was thought that vitamin E ensured separate transport of oxygen in silicone-rich channels in the hydrogels and transport of cysteamine in the hydrophilic channels. $^{52}$

A more advanced hydrogel polymer called nanowafers, which are transparent circular discs with a refractive index similar to that of soft contact lenses and malleable to the ocular surface, were also investigated as a vehicle for cysteamine. ${ }^{55}$ Cysteamine was converted to cystamine on nanowafers made of carboxymethyl cellulose, polyvinylpyrrolidone, and HPMC within 2 weeks. ${ }^{55}$ Conversely, nanowafers made with poly(vinyl alcohol) kept cysteamine in its active form for 4 months at room temperature and only dimerized to cystamine after 6 months. ${ }^{55}$ When compared to instillation of cysteamine $0.44 \%(44 \mu \mathrm{g})$ drops twice per day to a nanowafer with cysteamine $10 \mu \mathrm{g}$ once per day, 
the latter was more effective at clearing up cystine crystals by $65 \%$, compared to $34 \%$ with the drops. ${ }^{55}$ The additional advantages of nanowafers are that they self-disintegrate after 4-5 hours, are safe, and have lubricating properties on the ocular surface..$^{55}$

\section{Efficacy studies, including any relevant case reports}

Shams et al described a summary of treatment modalities available for corneal cystine crystal deposition. ${ }^{56}$ This review summarizes the efficacy studies, including case reports, of cysteamine hydrochloride drops in chronological order (Table 1).

Kaiser-Kupfer et al carried out a double-blind, randomized, placebo-controlled trial between November 1985 and September 1989 investigating 25 patients with cystinosis. ${ }^{57}$ They assigned patients into two groups: Group 1 comprised those younger than age 4 and Group 2 included patients between ages 4 and 31. ${ }^{57}$ They investigated instillation of $0.1 \%$ cysteamine in normal saline (prepared by National Institutes of Health Clinical Center's Pharmaceutical Development Service) in one eye, compared to placebo of normal saline in the second eye, instilled hourly during waking hours, with new bottles dispensed every 5 days. ${ }^{57}$ The cysteamine concentration was changed to $0.5 \%$ during the trial for some patients following animal studies and subsequent approval for use in patients. ${ }^{57}$ Investigators blinded to study allocation determined an end point as a difference in the "corneal density score" between the two eyes and a decrease in the "corneal density score" in the "better" eye compared to the previous visit. ${ }^{57}$ This corneal cystine crystal score included arbitrary units from 0 to 3.00 , with 0.25 increments, by subjectively comparing to a library of standard corneal transparency slides. ${ }^{57}$ Of the 16 patients in Group 1 , $50 \%$ showed a reduction in corneal cystine crystal score in the eye receiving both $0.1 \%$ or $0.5 \%$ cysteamine drops and the median time to the end point was 8 months. ${ }^{57} \mathrm{Of}$ the nine patients in Group 2, two patients receiving $0.5 \%$ cysteamine reached the end point at 6 and 9 months and the study elucidated that compliance was better in these patients than in patients not reaching the end point. ${ }^{57}$ This study concluded that, possibly, early administration of cysteamine drops at a young age would have a more beneficial effect on corneal cystine crystal clearance than at older ages. ${ }^{57}$

A subsequent case report from 1991 is of a 2-year-old with nephropathic cystinosis treated with $0.5 \%$ cysteamine drops hourly during waking hours in one eye. ${ }^{58}$ At 3 months, the cornea, especially in the center, had significantly less cystine crystals..$^{58}$ The untreated eye remained status quo,

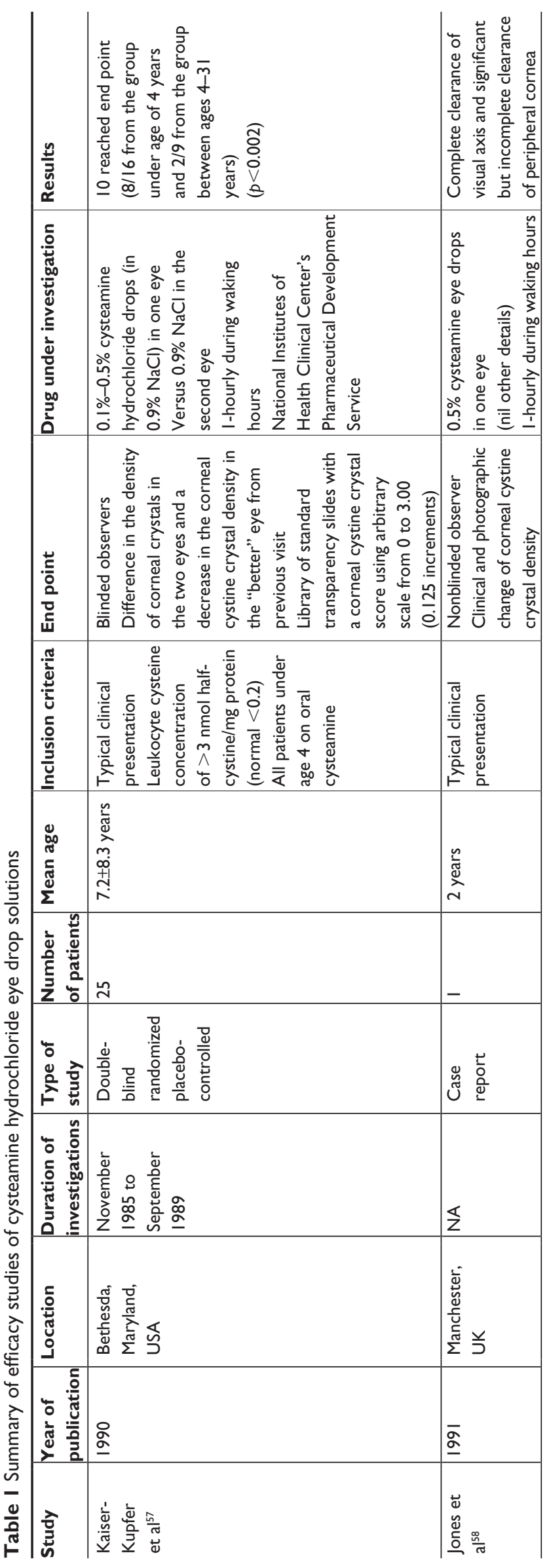




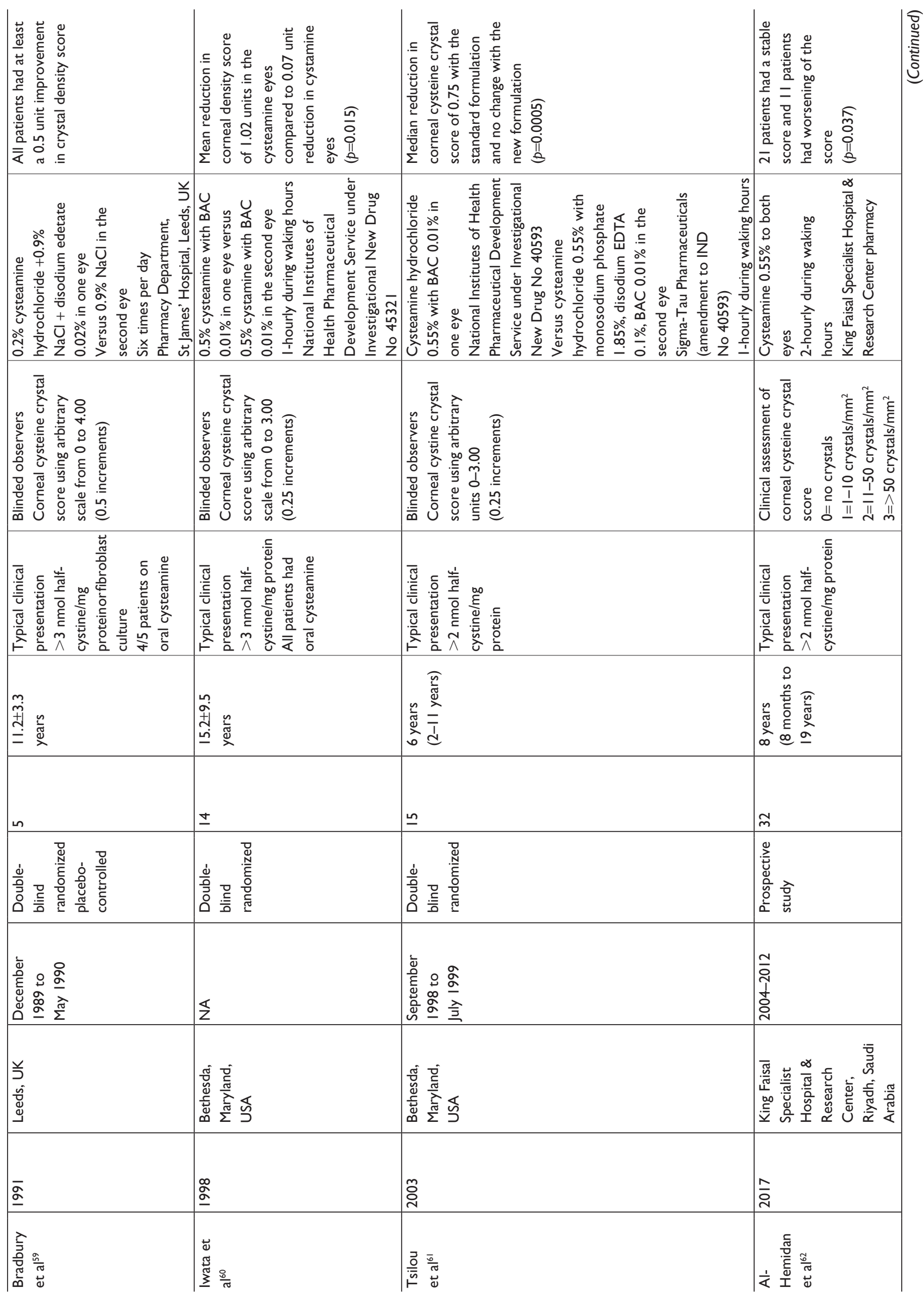




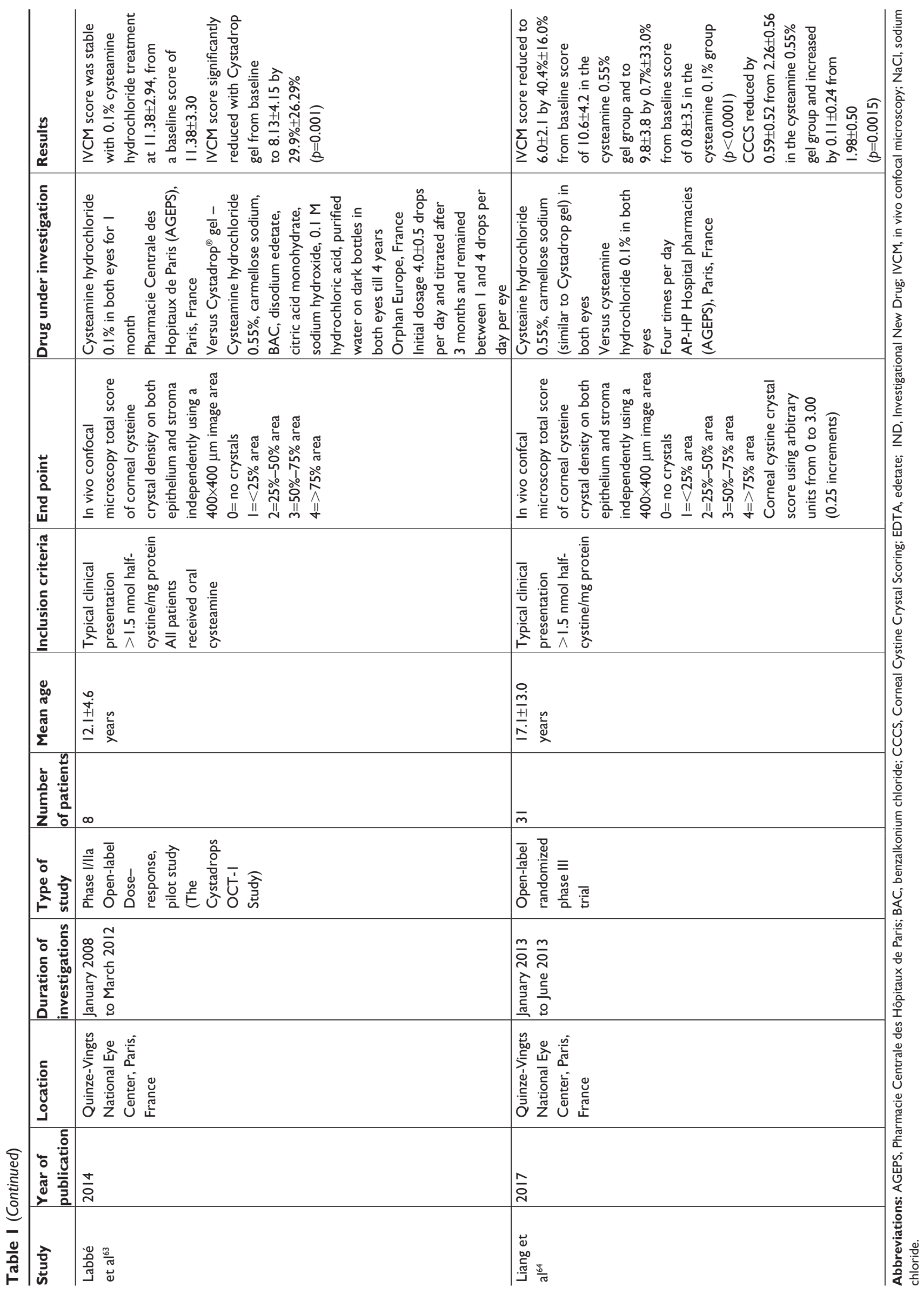


but was treated with $0.5 \%$ cysteamine drops thereafter and showed similar advancement in corneal cystine crystal reduction. ${ }^{58}$

Bradbury et al carried out another double-blind, randomized, placebo-controlled study on five patients with cystinosis between December 1989 and May 1990..$^{59}$ Patients received $0.2 \%$ cysteamine hydrochloride with disodium edetate $0.02 \%$ in normal saline (prepared by Pharmacy Department, St James' Hospital, Leeds, UK) in one eye, and the other eye received normal saline six times per day. ${ }^{59}$ Two observers determined the corneal cystine crystal score (on an arbitrary scale from 0 to 4.00 , with increments of 0.5 ) of slit-lamp photographs of corneas and were blinded to the treatment administered to that particular cornea. ${ }^{59}$ The corneal cystine crystal score improved in the treated eye in all patients by a mean score of 0.80 arbitrary units over 6 months. ${ }^{59}$

Furthermore, Iwata et al carried out a double-blind, randomized trial to compare cysteamine $0.5 \%$ with $\mathrm{BAC}$ drops, using the unstable cystamine $0.5 \%$ with BAC drops (prepared by National Institutes of Health Pharmaceutical Development Service under Investigational New Drug [IND] Exemption No 45321) in patients with cystinosis. ${ }^{60}$ Both drugs were frozen prior to use.$^{60}$ Half of the patients showed a reduction in the corneal cystine crystal score at first follow-up at 6-8 months in the eyes receiving cysteamine $0.5 \%$ drops. $^{60}$ Mean decrease in the corneal cystine crystal score was 1.02 units in the cysteamine $0.5 \%$ group, which was significantly greater than the reduction of the corneal cystine crystal score by 0.07 units in the cystamine $0.5 \%$ group. ${ }^{60}$

Tsilou et al carried out two prospective double-blind, randomized controlled trials investigating the efficacy of cysteamine hydrochloride $0.55 \%$ with $\mathrm{BAC} 0.01 \%$ in one eye of 15 patients (prepared by the National Institutes of Health Pharmaceutical Development Service under IND No 40593 ) and a new formulation (same as US FDA-approved $0.44 \%$ cysteamine from Sigma-Tau Pharmaceuticals without accounting for the moisture content of the hydrochloride) containing cysteamine hydrochloride $0.55 \%$ with monosodium phosphate $1.85 \%$, disodium EDTA $0.1 \%$, and BAC $0.01 \%$ (prepared by Sigma-Tau Pharmaceuticals as an amendment to IND No 40593) in the second eye, administered hourly during waking hours..$^{50,61}$ The drug was kept frozen, thawed before use, and kept at room temperature for 1 week only. ${ }^{61}$ A new frozen bottle was used each week. ${ }^{61}$ The corneal cystine crystal score showed at least one arbitrary unit of improvement (ranging from central clarity to greatest cysteine crystal density) in $47 \%$ receiving the standard treatment and no change in the patients receiving the new formulation. ${ }^{61}$ Authors suggested that the latter outcome might be secondary to the more frequently reported stinging and burning sensation with the new formulation, resulting in reflex blinking and elimination of the drug from the corneal surface prior to absorption. ${ }^{61}$ Moreover, a prospective study on 32 patients with nephropathic cystinosis had 2-hourly treatment with cysteamine $0.55 \%$ drops to both eyes (prepared by King Faisal Specialist Hospital \& Research Center pharmacy, Riyadh, Saudi Arabia). ${ }^{62}$ New bottles were used every 10 days. ${ }^{62}$ Approximately, two-thirds of the patients did not show a significant change in the corneal cystine crystal score and the other one-third of the patients had a significant increase of the corneal crystal score over a mean follow-up period of 4 years. ${ }^{62}$ The authors suggested that this might have been secondary to dissolution of cysteamine eye drops with blinking, poor absorption of drug, low concentration of drug used, or high density of cystine crystals on the cornea. ${ }^{62}$

In contrast, Labbé et al carried out a phase I/IIa trial, which was an open-label, dose-response, nonrandomized pilot study called The Cystadrops OCT-1 study, over 2008 and 2012, in France. ${ }^{63}$ They treated eight patients with infantile cystinosis with $0.1 \%$ cysteamine hydrochloride (prepared by Pharmacie Centrale des Hopitaux de Paris, France) in both eyes with a mean of $4.0 \pm 0.5$ drops per day for the first 30 days. ${ }^{63}$ At this point, treatment in both eyes was switched to mercaptamine, Cystadrops gel (cysteamine hydrochloride $0.55 \%$, carmellose sodium, BAC, disodium edetate, citric acid monohydrate, sodium hydroxide, $0.1 \mathrm{M}$ hydrochloric acid, and purified water in dark bottles prepared by Orphan Europe, France) at the same frequency and titrated at 3 months. ${ }^{63}$ In case of an increase in cystine crystal density, treatment was either stopped or frequency of drug administration was increased by one instillation. ${ }^{63}$ In contrast, the frequency of drug administration was reduced by one instillation if the cystine crystal density was stable and reduced by two instillations if an improvement was observed. ${ }^{63}$ At subsequent visits after 3 months, the frequency of drug administration was increased by one dose if the cystine crystal density showed an increase, continued at the same frequency if the disease process was stable, and reduced by one instillation if an improvement in the crystal density was observed..$^{63}$ New bottles that were kept in the fridge were used every week and, once opened, kept at room temperature during the daytime and refrigerated at night. ${ }^{63}$ In vivo confocal microscopy (IVCM) was used to quantify the crystal density over a $400 \times 400 \mu \mathrm{m}$ area of the central cornea on both the epithelium and stroma independently and classified as 0 , if no crystals; 1 , if covering $<25 \%$; 2 , if $25 \%-50 \%$; 3 , if $50 \%-75 \%$; and 4 , if $>75 \%$ of the defined area. ${ }^{63}$ The IVCM score remained stable over the first month while on $0.1 \%$ 
cysteamine hydrochloride drops. ${ }^{63}$ However, the IVCM score significantly reduced at 3 months after starting mercaptamine gel treatment and remained stable thereafter for a duration of 4 years of active treatment. ${ }^{63}$ This decrease was significant compared to the baseline at 4 years, even with down-titration of the treatment to a mean $3.0 \pm 1.07$ drops per day. ${ }^{63}$ When comparing layers of the cornea, the IVCM score of the epithelium reduced at 1 month $(0.1 \%$ cysteamine chloride) and at 3 months (mercaptamine gel), compared to the baseline. ${ }^{63}$ The IVCM score continued to decrease with mercaptamine gel, especially in the anterior stroma, even with reductions in frequency of instillations. ${ }^{63}$ The same group successively carried out an open-label phase III randomized trial comparing cysteamine hydrochloride $0.55 \%$ gel (equivalent to mercaptamine $0.55 \%$, Orphan Europe, France) with carmellose sodium stored in dark vials that were renewed weekly and $0.1 \%$ cysteamine hydrochloride drops (prepared by Pharmacie Centrale des Hôpitaux de Paris [AGEPS], AP-HP Hospital Pharmacies, Paris, France) four times per day to both eyes. ${ }^{64}$ They had 15 patients in the cysteamine $0.55 \%$ gel group and 16 in the cysteamine $0.1 \%$ group. ${ }^{64}$ The mean IVCM score reduced significantly from $10.6 \pm 4.2$ at baseline to $6.0 \pm 2.1$ at 3 months in the cysteamine $0.55 \%$ gel group, and from $10.8 \pm 3.5$ to $9.8 \pm 3.8$ in the cysteamine $0.1 \%$ group. ${ }^{64}$ The absolute reduction of $4.6 \pm 3.1$ in the cysteamine $0.55 \%$ gel group was significantly greater than the absolute reduction of $0.5 \pm 3.4$ in the cysteamine $0.1 \%$ group. ${ }^{64}$ The IVCM scores were considerably lower in most corneal layers in eyes treated with cysteamine $0.55 \%$ gel after 1 month. ${ }^{64}$ The mean corneal cysteine crystal score reduced by $0.59 \pm 0.52$ from a baseline score of $2.26 \pm 0.56$ in the cysteamine $0.55 \%$ gel group at 3 months, and this reduction was not apparent with cysteamine $0.1 \%{ }^{64}$ This increase in efficacy was owed to the increase in residence time of the $0.55 \%$ cysteamine hydrochloride gel and, therefore, to its ability to penetrate deeper layers of the cornea. ${ }^{64}$ This preparation of cysteamine hydrochloride $0.55 \%$ gel acquired European Marketing Authorization in January 2017. ${ }^{64}$ Cysteamine hydrochloride $0.1 \%$ has now been removed from the market due to its failure to show efficacy in treating corneal cystine crystals. ${ }^{65}$

\section{Safety and tolerability}

None of the studies reported any serious adverse events. These were described to be vision loss, severe redness expanding over $50 \%$ of the conjunctiva, and severe pain affecting daily activities. ${ }^{61}$ Transient local adverse events lasting less than 1 hour were described in most studies. These were namely, stinging, burning, and redness and were likely due to increased viscosity; therefore, increased residence time as well as a higher concentration of cysteamine hydrochloride were implicated as causes. ${ }^{63,64}$ A tolerability questionnaire in the form of Comparison of Ophthalmic Medications for Tolerability was completed by five patients in the phase III trial described above and, at 90 days, three were "very satisfied" and two were "somewhat satisfied" with cysteamine $0.55 \%$ gel treatment. ${ }^{64}$ All five patients expressed their preference toward the new treatment compared to their previous treatment. ${ }^{64}$

\section{Patient-focused perspectives, such} as quality of life, patient satisfaction/ acceptability, adherence, and follow-up

Published data on patient-focused perspectives are limited. Compliance, even at 1-hourly instillations, was generally good. Adherence was further shown to improve with counseling. ${ }^{57}$

Impact of photophobia, from cystine crystals likely stimulating sensory nerves at the basal epithelium and stroma, was considered in most studies. ${ }^{57}$ This is expected because a symptom, such as photophobia, will notably affect a patient's quality of life. In most studies, there was a parallel reduction in both subjective photophobia and clinician-assessed photophobia with corneal cystine crystal density when cysteamine hydrochloride $0.55 \%$ topical treatment was administered, which led to younger patients being able to play outdoors and even observe sunsets. ${ }^{60,64}$ Furthermore, some studies reported an improvement in visual acuity and contrast sensitivity with cysteamine hydrochloride $0.55 \% .^{59,64}$

\section{Conclusion, place of cysteamine hydrochloride eye drop solutions as a treatment modality for corneal cystine crystal deposition}

Given that cystinosis is an orphan disease, there are only a few reports studying effects of cysteamine hydrochloride topical treatment in the treatment of corneal cystine crystals. This is a significant reason for various end points studied over the course of the last 30 years. In addition, the availability of more advanced technology has allowed researchers to measure corneal cystine crystals both quantitatively and qualitatively, such as with in vivo confocal microscopy. Although in vivo confocal microscopy may be the best method so far in quantifying crystal density and describing its distribution in each layer of the cornea, it may not be readily available in all eye units. Clinical assessment, as described by Al-Hemidan et al, ${ }^{62}$ where the number of crystals per square millimeter is defined, may also be a suitable objective method 
of quantifying these crystals. However, determining visual acuity and level of photophobia may be the most relevant method of determining the effect of treatment on the patient's quality of life.

This review shows that topical cysteamine hydrochloride at $0.55 \%$ is efficacious as well as safe in treating corneal cystine crystals. These objective improvements in corneal cystine crystal density reduce the risk of complications, such as corneal erosions, scarring, and neovascularization. ${ }^{57}$ Furthermore, they can be associated with subjective improvements in photophobia and, thence, quality of life. ${ }^{57}$ However, treatment in the form of drops requiring frequent administration is likely to lead to poor adherence and burden on carers who have to instill the treatment to patients who are largely a pediatric population. More viscous therapy in the form of gels instilled less regularly can be just as efficacious, but more studies utilizing contact lenses and nanowafers described herein can be valuable. Moreover, this expensive drug has practicality issues with regard to being unstable at room temperature and having a short half-life of 1 week once opened. Finally, cystine crystals can also be deposited in the iris and ciliary body causing complications, such as glaucoma. However, topical cysteamine was not shown to reach significant drug levels in the aqueous humor in vivo and, therefore, this should be considered in future studies. ${ }^{66}$

\section{Disclosure}

The authors report no conflicts of interest in this work.

\section{References}

1. Gahl WA, Bashan N, Tietze F, Bernardini I, Schulman JD. Cystine transport is defective in isolated leukocyte lysosomes from patients with cystinosis. Science. 1982;217(4566):1263-1265.

2. Gahl WA, Thoene JG, Schneider JA. Cystinosis. $N$ Engl J Med. 2002;347(2):111-121.

3. Kalatzis V, Antignac C. New aspects of the pathogenesis of cystinosis. Pediatr Nephrol. 2003;18(3):207-215.

4. Gahl WA, Balog JZ, Kleta R. Nephropathic cystinosis in adults: natural history and effects of oral cysteamine therapy. Ann Intern Med. 2007;147(4):242-250.

5. Wilmer MJ, Emma F, Levtchenko EN. The pathogenesis of cystinosis: mechanisms beyond cystine accumulation. Am J Physiol Renal Physiol. 2010;299(5):F905-F916.

6. Forestier L, Jean G, Attard M, et al. Molecular characterization of CTNS deletions in nephropathic cystinosis: development of a PCR-based detection assay. Am J Hum Genet. 1999;65(2):353-359.

7. Touchman JW, Anikster Y, Dietrich NL, et al. The genomic region encompassing the nephropathic cystinosis gene (CTNS): complete sequencing of a 200-kb segment and discovery of a novel gene within the common cystinosis-causing deletion. Genome Res. 2000;10(2):165-173.

8. Town M, Jean G, Cherqui S, et al. A novel gene encoding an integral membrane protein is mutated in nephropathic cystinosis. Nat Genet. 1998;18(4):319-324.
9. Elmonem MA, Veys KR, Soliman NA, van Dyck M, van den Heuvel LP, Levtchenko E. Cystinosis: a review. Orphanet J Rare Dis. 2016; $11: 47$.

10. Shotelersuk V, Larson D, Anikster Y, et al. CTNS mutations in an American-based population of cystinosis patients. Am J Hum Genet. 1998;63(5):1352-1362.

11. Kalatzis V, Cherqui S, Jean G, et al. Characterization of a putative founder mutation that accounts for the high incidence of cystinosis in Brittany. J Am Soc Nephrol. 2001;12(10):2170-2174.

12. McGowan-Jordan J, Stoddard K, Podolsky L, et al. Molecular analysis of cystinosis: probable Irish origin of the most common French Canadian mutation. Eur J Hum Genet. 1999;7(6):671-678.

13. Elmonem MA, Mahmoud IG, Mehaney DA, et al. Lysosomal storage disorders in Egyptian children. Indian J Pediatr. 2016;83(8): 805-813.

14. Topaloglu R, Vilboux T, Coskun T, et al. Genetic basis of cystinosis in Turkish patients: a single-center experience. Pediatr Nephrol. 2012;27(1):115-121.

15. Anikster Y, Lucero C, Touchman JW, et al. Identification and detection of the common $65-\mathrm{kb}$ deletion breakpoint in the nephropathic cystinosis gene (CTNS). Mol Genet Metab. 1999;66(2):111-116.

16. Attard M, Jean G, Forestier L, et al. Severity of phenotype in cystinosis varies with mutations in the CTNS gene: predicted effect on the model of cystinosin. Hum Mol Genet. 1999;8(13):2507-2514.

17. Kalatzis V, Nevo N, Cherqui S, Gasnier B, Antignac C. Molecular pathogenesis of cystinosis: effect of CTNS mutations on the transport activity and subcellular localization of cystinosin. Hum Mol Genet. 2004;13(13):1361-1371.

18. Tsilou E, Zhou M, Gahl W, Sieving PC, Chan CC. Ophthalmic manifestations and histopathology of infantile nephropathic cystinosis: report of a case and review of the literature. Surv Ophthalmol. 2007; 52(1):97-105.

19. Thoene JG. A review of the role of enhanced apoptosis in the pathophysiology of cystinosis. Mol Genet Metab. 2007;92(4):292-298.

20. Bois E, Feingold J, Frenay P, Briard ML. Infantile cystinosis in France: genetics, incidence, geographic distribution. J Med Genet. 1976;13(6): 434-438.

21. Aldahmesh MA, Humeidan A, Almojalli HA, et al. Characterization of CTNS mutations in Arab patients with cystinosis. Ophthalmic Genet 2009;30(4):185-189.

22. Soliman NA, Bazaraa HM, Abdel Hamid RH, Badawi N. Nephropathic cystinosis in a developing country: the Egyptian experience. Saudi J Kidney Dis Transpl. 2013;24(1):147-149.

23. Kitnarong N, Osuwannaratana P, Kamchaisatian W, Namtongthai P, Metheetrairut A. Ocular manifestations in adolescent cystinosis: case report in Thailand. J Med Assoc Thai. 2005;88(4):521-526.

24. Hutchesson AC, Bundey S, Preece MA, Hall SK, Green A. A comparison of disease and gene frequencies of inborn errors of metabolism among different ethnic groups in the West Midlands, UK. J Med Genet. 1998;35(5):366-370.

25. De Braekeleer M. Hereditary disorders in Saguenay-Lac-St-Jean (Quebec, Canada). Hum Hered. 1991;41(3):141-146.

26. Manz F, Gretz N. Cystinosis in the Federal Republic of Germany. Coordination and analysis of the data. J Inherit Metab Dis. 1985; $8(1): 2-4$.

27. Meikle PJ, Hopwood JJ, Clague AE, Carey WF. Prevalence of lysosomal storage disorders. JAMA. 1999;281(3):249-254.

28. Hult M, Darin N, von Döbeln U, Månsson JE. Epidemiology of lysosomal storage diseases in Sweden. Acta Paediatr. 2014;103(12): 1258-1263.

29. Ebbesen F, Mygind KI, Holck F. Infantile nephropatic cystinosis in Denmark. Dan Med Bull. 1976;23(5):216-222.

30. Nakhaii S, Hooman N, Otukesh H. Gastrointestinal manifestations of nephropathic cystinosis in children. Iran J Kidney Dis. 2009;3(4): 218-221.

31. Vaisbich MH, Koch VH. Report of a Brazilian multicenter study on nephropathic cystinosis. Nephron Clin Pract. 2010;114(1):c12-c18. 
32. Kaiser-Kupfer MI, Caruso RC, Minkler DS, Gahl WA. Long-term ocular manifestations in nephropathic cystinosis. Arch Ophthalmol. 1986; 104(5):706-711.

33. Tsilou ET, Rubin BI, Reed GF, Iwata F, Gahl W, Kaiser-Kupfer MI. Age-related prevalence of anterior segment complications in patients with infantile nephropathic cystinosis. Cornea. 2002;21(2):173-176.

34. Kocabora MS, Ozbilen KT, Altunsoy M, Ahishali B, Taskapili M. Clinicopathological features of ocular cystinosis. Clin Exp Ophthalmol. 2008;36(8):778-781.

35. Bishop R. Ocular complications of infantile nephropathic cystinosis. J Pediatr. 2017;183S:S19-S21.

36. Tsilou E, Rubin BI, Reed G, et al. Nephropathic cystinosis: posterior segment manifestations and effects of cysteamine therapy. Ophthalmology. 2006;113(6):1002-1009.

37. Katz B, Melles RB, Schneider JA. Contrast sensitivity function in nephropathic cystinosis. Arch Ophthalmol. 1987;105(12):1667-1669.

38. Gretz N, Manz F, Augustin R, et al. Survival time in cystinosis. A collaborative study. Proc Eur Dial Transplant Assoc. 1983;19:582-589.

39. Almond PS, Matas AJ, Nakhleh RE, et al. Renal transplantation for infantile cystinosis: long-term follow-up. J Pediatr Surg. 1993;28(2): 232-238.

40. Van Stralen KJ, Emma F, Jager KJ, et al. Improvement in the renal prognosis in nephropathic cystinosis. Clin J Am Soc Nephrol. 2011;6(10): 2485-2491.

41. Spicer RA, Clayton PA, McTaggart SJ, Zhang GY, Alexander SI. Patient and graft survival following kidney transplantation in recipients with cystinosis: a cohort study. Am J Kidney Dis. 2015;65(1):172-173.

42. Lewis MA, Shaw J, Sinha M, Adalat S, Hussain F, Inward C. UK Renal Registry 11th Annual Report (December 2008): Chapter 13 Demography of the UK paediatric renal replacement therapy population. Nephron Clin Pract. 2009;111(Suppl 1):c257-c267.

43. Cohen C, Charbit M, Chadefaux-Vekemans B, et al. Excellent longterm outcome of renal transplantation in cystinosis patients. Orphanet J Rare Dis. 2015;10:90.

44. Bertholet-Thomas A, Bacchetta J, Tasic V, Cochat P. Nephropathic cystinosis - a gap between developing and developed nations. $N$ Engl J Med. 2014;370(14):1366-1367.

45. Kaiser-Kupfer MI, Fujikawa L, Kuwabara T, Jain S, Gahl WA. Removal of corneal crystals by topical cysteamine in nephropathic cystinosis. N Engl J Med. 1987;316(13):775-759.

46. Katz B, Melles RB, Schneider JA. Crystal deposition following keratoplasty in nephropathic cystinosis. Arch Ophthalmol. 1989; 107(12):1727-1728

47. Simpson JL, Nien CJ, Flynn KJ, Jester JV. Evaluation of topical cysteamine therapy in the CTNS(-/-) knockout mouse using in vivo confocal microscopy. Mol Vis. 2011;17:2649-2654.

48. Thoene JG, Oshima RG, Crawhall JC, Olson DL, Schneider JA. Cystinosis. Intracellular cystine depletion by aminothiols in vitro and in vivo. J Clin Invest. 1976;58(1):180-189.

49. Pescina S, Carra F, Padula C, Santi P, Nicoli S. Effect of pH and penetration enhancers on cysteamine stability and trans-corneal transport. Eur J Pharm Biopharm. 2016;107:171-179.

50. Huynh N, Gahl WA, Bishop RJ. Cysteamine ophthalmic solution 0.44\% for the treatment of corneal cystine crystals in cystinosis. Expert Rev Ophthalmol. 2013;8(4):341-345.

Clinical Ophthalmology

\section{Publish your work in this journal}

Clinical Ophthalmology is an international, peer-reviewed journal covering all subspecialties within ophthalmology. Key topics include: Optometry; Visual science; Pharmacology and drug therapy in eye diseases; Basic Sciences; Primary and Secondary eye care; Patient Safety and Quality of Care Improvements. This journal is indexed on Submit your manuscript here: http://www.dovepress.com/clinical-ophthalmology-journal
51. McKenzie B, Kay G, Matthews KH, Knott R, Cairns D. Preformulation of cysteamine gels for treatment of the ophthalmic complications in cystinosis. Int J Pharm. 2016;515(1-2):575-582.

52. Hsu KH, Fentzke RC, Chauhan A. Feasibility of corneal drug delivery of cysteamine using vitamin $\mathrm{E}$ modified silicone hydrogel contact lenses. Eur J Pharm Biopharm. 2013;85(3 Pt A):531-540.

53. Bozdağ S, Gümüş K, Gümüş $\mathrm{O}$, Unlü N. Formulation and in vitro evaluation of cysteamine hydrochloride viscous solutions for the treatment of corneal cystinosis. Eur J Pharm Biopharm. 2008;70(1):260-269.

54. Buchan B, Kay G, Heneghan A, Matthews KH, Cairns D. Gel formulations for treatment of the ophthalmic complications in cystinosis. Int J Pharm. 2010;392(1-2):192-197.

55. Marcano DC, Shin CS, Lee B, et al. Synergistic cysteamine delivery nanowafer as an efficacious treatment modality for corneal cystinosis. Mol Pharm. 2016;13(10):3468-3477.

56. Shams F, Livingstone I, Oladiwura D, Ramaesh K. Treatment of corneal cystine crystal accumulation in patients with cystinosis. Clin Ophthalmol. 2014;8:2077-2084.

57. Kaiser-Kupfer MI, Gazzo MA, Datiles MB, Caruso RC, Kuehl EM, Gahl WA. A randomized placebo-controlled trial of cysteamine eye drops in nephropathic cystinosis. Arch Ophthalmol. 1990;108(5):689-693.

58. Jones NP, Postlethwaite RJ, Noble JL. Clearance of corneal crystals in nephropathic cystinosis by topical cysteamine $0.5 \%$. Br J Ophthalmol. 1991 ; 75(5):311-312.

59. Bradbury JA, Danjoux JP, Voller J, Spencer M, Brocklebank T. A randomised placebo-controlled trial of topical cysteamine therapy in patients with nephropathic cystinosis. Eye (Lond). 1991;5(Pt 6): 755-760.

60. Iwata F, Kuehl EM, Reed GF, McCain LM, Gahl WA, Kaiser-Kupfer MI. A randomized clinical trial of topical cysteamine disulfide (cystamine) versus free thiol (cysteamine) in the treatment of corneal cystine crystals in cystinosis. Mol Genet Metab. 1998;64(4):237-242.

61. Tsilou ET, Thompson D, Lindblad AS, et al. A multicentre randomised double masked clinical trial of a new formulation of topical cysteamine for the treatment of corneal cystine crystals in cystinosis. Br JOphthalmol. 2003;87(1):28-31.

62. Al-Hemidan A, Shoughy SS, Kozak I, Tabbara KF. Efficacy of topical cysteamine in nephropathic cystinosis. Br J Ophthalmol. 2017; 101(9):1234-1237.

63. Labbé A, Baudouin C, Deschênes G, et al. A new gel formulation of topical cysteamine for the treatment of corneal cystine crystals in cystinosis: the Cystadrops OCT-1 study. Mol Genet Metab. 2014;111(3): 314-320.

64. Liang H, Labbé A, Le Mouhaër J, Plisson C, Baudouin C. A new viscous cysteamine eye drops treatment for ophthalmic cystinosis: an open-label randomized comparative phase III pivotal study. Invest Ophthalmol Vis Sci. 2017;58(4):2275-2283.

65. www.ema.europa.eu/ema [homepage on the Internet]. Assessment report: Dropcys. Available from: http://www.ema.europa.eu/docs/ en_GB/document_library/EPAR_-_Public_assessment_report/ human/004038/WC500210937.pdf. Accessed October 7, 2017.

66. Hsuan JD, Harding JJ, Bron AJ. The penetration of topical cysteamine into the human eye. J Ocul Pharmacol Ther. 1996;12(4):499-502.
PubMed Central and CAS, and is the official journal of The Society of Clinical Ophthalmology (SCO). The manuscript management system is completely online and includes a very quick and fair peer-review system, which is all easy to use. Visit http://www.dovepress.com/ testimonials.php to read real quotes from published authors. 